\title{
THE TRYPANOSOMES OF TSETSE FLIES.*
}

FREDERICK G. NOVY.

(From the Hygienic Laboratory, University of Michigan, Ann Arbor, Mich.)

INFLUENCED by the studies of Schaudinn regarding the presence of trypanosomes in the gut of mosquitoes, many workers have endeavored to apply the same method of investigation to the solution of related problems. Thus, the trypanosomes and trypanoplasmas of fish have been traced to the flagellates in the intestine of the leech; the trypanosome of the rat to like flagellates present in the louse; and, more recently, Tr. gambiense, the cause of sleeping sickness, and Tr. Brucei, that of nagana, have been ascribed a direct relationship to the flagellates present in the stomach of the tsetse fly.

Inasmuch as all of our work during the past two years has shown that the results of Schaudinn are open to an entirely different conclusion from that arrived at by him, and quite generally accepted by others, it was of interest to examine into the supposed relationship of the two pathogenic trypanosomes mentioned above to the flagellated forms present in the stomach of the flies. The results obtained in our studies of the flagellates of mosquitoes $\dagger$ justified the belief that intestinal parasitism due to relatively harmless flagellates would be found to be a common natural condition.

In this examination we were not confined to a mere critical study of the published papers dealing with this subject, but through the kindness of Lieutenant A. C. H. Gray, R.A., M.C., member of the Sleeping Sickness Commission, we received a number of smears from six tsetse flies (Glossina palpalis) captured at Entebbe, Uganda, Central Africa. All of these contained trypanosomes, some in very large numbers, and consequently it was possible at first hand to make a microscopic and photographic study of the organisms. It is a pleasure, therefore, to acknowledge this very great courtesy of Lieutenant Gray, without whose aid it would have been quite impossible to arrive at definite conclusions.

* Received for publication March 14, Igo6.

† Novy, MacNeal, and Torrey, "Mosquito Trypanosomes." An abstract of the paper read before the Society of American Bacteriologists, Dec, 28, rgo5. Appeared in Science, Feb. 9, 1906, p. 207. 
Before taking up the description of the trypanosomes present in the slides sent by Dr. Gray, it will be desirable to review at length the publications which have already been made on this subject. This is all the more necessary since a mere consideration of these papers will show how readily erroneous conclusions may be drawn from otherwise simple facts.

In order to ascertain whether the trypanosome of sleeping sickness could multiply in the stomach of Glossina palpalis, Gray and Tulloch ${ }^{1}$ fed flies which had been caught 24 to 48 hours previously, on monkeys infected from the cerebrospinal fluid of sleeping-sickness cases. The flies were refed on infected monkeys every 48 hours.

An examination of the flies 96 and 120 , and even as early as 24 hours after feeding showed that the blood in the intestines literally swarmed with trypanosomes. By successive feeding on blood the flies were found to maintain the trypanosomes in greatly increased numbers up to 12 days after the infective feed. It is significant that Io per cent of all the flies, examined 24 hours after feeding, showed this multiplication of trypanosomes. The authors do not commit themselves to the extent of regarding these trypanosomes as actual multiplication forms of Tr. gambiense. They noted the very suspicious fact that on some days a considerable number of flies would be examined with negative results, although they were kept under the same conditions and fed on the same monkeys as at other times when successful results were obtained.

The appearance of the trypanosomes within 24 hours after feeding and the further important fact that many batches of flies failed to show any trypanosomes on identical feeding, led to an examination of freshly caught flies which were fed on an uninfected monkey. It was found that two out of 200 flies examined contained the same enormous numbers as those found in the flies which fed on infected monkeys. In other words, I per cent of the flies fed on uninfected monkeys, while Io per cent of those fed on infected monkeys, showed trypanosomes. Since then infected flies have been met with more often, for the specimens which we have studied came from flies which had not even been fed on animals. In his letter Dr. Gray makes

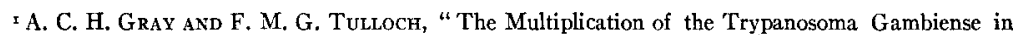
the Alimentary Canal of Glossina Palpalis," Reports of the Sleeping Sickness Commission, of the Roya Society, No. VI, August, 1905, pp. $282^{-87}$. Four figures. Harrison \& Sons, London. 
the very significant statement that 8 per cent of the freshly caught flies from the uninhabited island of Kimmi contained these flagellates, while only 2 per cent of those taken in Entebbe were infected.

Extremely important as bearing upon the nature of the fly trypanosome is the fact that not one of five monkeys which received injections of enormous numbers of these flagellates developed an infection. The failure to produce an infection clearly indicates that the trypanosomes are not derived from the Tr. gambiense of the monkey's blood. The latter organism was never present in the blood of monkeys in larger number than one in six fields of the $2 \mathrm{~mm}$. objective, and the negative inoculation experiments simply indicate that the $T r$. gambiense which had been taken into the stomach of the fly had died off in the interval.

According to Gray and Tulloch, the trypanosomes seen in the fly vary from very small ones, some $20 \mu$ in length, to very long slender ones of about roo $\mu$. Attention was called to the position of the micronucleus, which was rarely seen at the extreme blunted end of the parasite. It was found to vary from a position midway between the posterior extremity of the trypanosome and the macronucleus, to a position on the anterior or flagellar side. They also noted the presence of divisional forms, as well as of rosettes, the members of which were joined directly by their posterior ends. These groups consisted of four to seven cells, or of large, loosely woven masses of 15 to 20 cells. The trypanosomes were found throughout the blood in the whole alimentary tract.

The trypanosomes of tsetse flies have further been studied by Dr. R. Koch, ${ }^{\mathrm{I}}$ who at first found them in Glossina fusca. Of 60 infected flies one was Gl. morsitans, and one was Gl. pallidipes, and for this reason he concludes that the tsetse fly disease in the German Protectorate is not exclusively carried by Gl. morsitans, but that it is also conveyed by Gl. pallidipes and chiefly by Gl. fusca. This conclusion, since it is based primarily upon the presence of flagellates in the flies, it will be seen is open to question. The percentage of infected flies is not given by him, except in he case of one locality, where the highest percentage (I7.4) was observed.

\footnotetext{
x. KocH, "Vorläufige Mitteilungen über die Ergeebniss einer Forschungsreise nach Ostafrika," Deutsche med. Wchnschr., Nov, 28, 1905, pp. 1865-69. 24 figures.
} 
He was unable to infect Glossinas by feeding them on animals which contained trypanosomes in their blood. When fed on cattle, the blood of which was rich in trypanosomes, the flagellates disappeared from the stomach as the digestion of the blood progressed; there was neither a development of trypanosomes nor a permanent infection of the flies as a result. He attaches particular importance to the fact that some flies were infected when they fed on animals, such as oxen and mules, which had but a few trypanosomes in their blood, and he assumes, therefore, that not all of the blood trypanosomes are adapted for the infection of flies, but only such as occur in a definite, but as yet unknown, stage.

It follows from the foregoing that most, if not all, of the 60 infected flies referred to above contained trypanosomes at the time of their capture, and hence these were acquired while at large, and not as the result of experimental feeding. He first noted the presence of trypanosomes in the clear droplet which exudes when pressure is applied to the bulb at the base of the proboscis. The absence in the fluid of blood corpuscles and the presence of the trypanosomes in very large numbers, and in divisional stages, led him to conclude that the flies did not transmit the disease by carrying the blood directly from an animal to another, as is usually supposed, but that, as in the case of the malarial parasite, the trypanosomes pass through a development stage in the flies. This assumption he believed to be verified by the fact that on examining the flies he found that the stomach contained large quantities of trypanosomes in various stages of development, even when the stomach was entirely free from blood.

Among the tsetse trypanosomes he recognized two forms; one thick and plump, the other long and slender. The former, which stained blue, and had a rather large round nucleus of loose texture, he regards as the female; while the latter type, which does not stain blue, and has a long, narrow, rod-shaped, and very compact nucleus, he considers as the male cell. Whether the two forms conjugate directly, or whether the latter gives off microgametes, he leaves undetermined, though he inclines to the latter view.

In the lower part of the stomach he found forms which suggested fertilized female trypanosomes. These were large cells which con- 
tained two, usually four, and at times eight, nuclei, although but one blepharoplast and one flagellum were present. This unusual condition of many nuclei and but one blepharoplast is certainly not the result of ordinary division where the division of the blepharoplast always precedes that of the nucleus.* $\mathrm{He}$ inclines to view it as a multiplication change consequent upon fertilization; the multinucleated trypanosomes eventually breaking up into many parts, thus giving rise to the youngest forms which first appear as round bodies with only a nucleus. Later these elongate to forms which have a nucleus and a blepharoplast, and these in turn develop a flagellum, and thus become true trypanosomes. Particularly important is the observation that rats injected with the fly trypanosomes failed to develop an infection.

In a second paper, ${ }^{\mathrm{I}}$ which appeared on the same date, Dr. Koch endeavors to establish differences between $T r$. Brucei and Tr. gambiense based upon morphological variations of the trypanosomes found in Glossina morsitans, Gl. fusca, and Gl. palpalis, the first two flies being the carriers of Tr. Brucei, while the latter is that of Tr. gambiense, the cause of sleeping sickness. He points out, as in the preceding paper, that in the infected flies the trypanosomes grow much larger than in the blood of animals, and that they present the two types, female and male, referred to above. A' comparison of the sexual forms of Tr. Brucei and Tr. gambiense showed differences which he thinks may be used to separate the organisms into two distinct kinds or species. The most important difference noted was the behavior of the blepharoplast in the female type. According to his statement the blepharoplast of $T r$. Brucei is small, round, and I. $O u$ in diameter; while that of $T r$ gambiense stains intensely and is surprisingly large, $1.5 \times 2.5 \mu$, is oval or rodlike, and always lies at righ ${ }^{\star}$ angles to the long axis of the trypanosome. A second point of differentiation-to which, however; he attaches less significance -is the size of the male types of both trypanosomes. For these he gives the following measurements:

\footnotetext{
*The occurrence of two or three nuclei with but one blepharoplast is not an uncommon feature in cultures of various trypanosomes. Thus, we have found such forms in cultures of Tr. Levisi, Crithidia fasciculata, and in those of several bird trypanosomes.

x "Über die Unterscheidung der Trypanosomenarten," Sitzungsberichte der königl. preus. Akademie der Wissenschaften, Nov. 23, 1905, pp. 957-62.
} 


\begin{tabular}{|c|c|c|c|}
\hline & & Length in $\mu$ & Width in $\mu$ \\
\hline $\left.\begin{array}{l}\text { Tr. Brucei } \\
\text { Tr. gambiense } \\
\text { Tr. Brucei } \\
\text { Tr. gambiense }\end{array}\right\}$ & 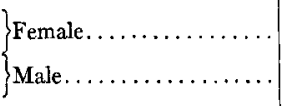 & $\begin{array}{l}25.0 \\
37.0 \\
40.2 \\
34.0\end{array}$ & $\begin{array}{l}3.60 \\
3.00 \\
2.10 \\
0.85\end{array}$ \\
\hline
\end{tabular}

With reference to the above it should be noted that the trypanosome present in Gl. palpalis is assumed by Dr. Koch to be Tr. gambiense, while that in Gl. fusca and Gl. morsitans was likewise assumed to be Tr. Brucei, notwithstanding no actual infection of flies by these organisms had been demonstrated, much less infection of animals by the bites of such flies, or by the injection of their stomach contents. Parenthetically it may be stated that a better and more certain means of differentiation is afforded, as we have repeatedly indicated, by the cultural characteristics of artificially grown, pure strains of trypanosomes. The marked differences presented by cultures of Tr. Lewisi, Tr. Brucei, Tr. Evansi, and of the various trypanosomes isolated from birds and mosquitoes, are sufficient evidence on this point, and it is somewhat surprising that the importance of the application of this method, so fundamental in bacteriology, does not seem to be recognized in protozoal work.

Having outlined at some length the observations made thus far on the trypanosomes of tsetse flies, it is in order to point out that, in our opinion, wholly erroneous conclusions have been drawn from the seen facts. It is our belief that the trypanosomes met with in the tsetse flies are "cultural" forms of harmless non-parasitic flagellates corresponding to the equally harmless Herpetomonas and Crithidia which we have found in mosquitoes. This view is based upon the following facts:

r. The large size of the trypanosomes as compared with that of the blood forms.

2. Their presence in flies which presumably had not fed on infected animals.

3. The failure to secure growths of the trypanosomes, pathogenic or non-pathogenic, in all, or even in a large per cent, of the flies fed on infected animals.

4. The failure to infect susceptible animals.

5. The analogy to mosquito trypanosomes. 
Under No. I. - It may be stated at the outset that an examination of the illustrations given by Gray and Tulloch, as well as those of Dr. Koch, suggests immediately that the trypanosomes in question are cultural types corresponding, in general, to the various forms which we have cultivated during the past three years. This view received definite confirmation during the subsequent study of the smears made from tsetse flies.

We have repeatedly called attention to the existence, in cultures of diverse trypanosomes, of two types quite unlike in many respects. A particularly good example of this duality of form is seen in the cultures of $T r$. avium which is characterized by multiplication rosettes and free-swimming threadlike spirochetes. The term "spirochete" must no longer be employed to designate this form, since, unlike the true spirochete, it possesses the typical structure of a trypanosome; that is to say, it has a nucleus, blepharoplast, undulating membrane, and a whip, all of which are easily demonstrated. Inasmuch as corresponding forms were met with in the blood, the view was expressed ${ }^{\mathrm{I}}$ that the large form of blood trypanosome gave rise, in cultures, to the rosette type and that it represented the female cell, while the small form gave rise to the spirochete-like type which may be considered as an indifferent or asexual form.

As indicated above, Dr. Koch regards the wide type met with in tsetse flies as the female, and the long narrow type as the male trypanosome. In this respect, then, they present a marked "cultural" characteristic. Another feature which indicates that the tsetse trypanosomes are cultural forms is seen in the position of the blepharoplast which usually lies anterior to the nucleus, exceptionally by the side or posterior to it. Again, in cultural forms the ratio of the width to the length of the cell is invariably less than that of the blood forms. Thus, while the ratio of the small blood form of $T r$. avium is about 5 to $20 \mu$, that of the corresponding spirochete-like type is I to $3^{\circ-60 \mu}$. In other words, the bulk or mass of the cultural trypanosome is always less than that of the blood form.

It may be taken as a general proposition that a given organism, bacterial or protozoal, when it undergoes rapid multiplication gives rise to smaller forms than it does under inverse conditions. And p. 287.

r F. G. Novy and W. J. MacNeal., "On the Trypanosomes of Birds," Jour. Infec. Dis., I905, 2 
since the tsetse trypanosomes, notwithstanding their great numbers, which indicate rapid multiplication, are much larger than the Tr. gambiense of human or mammalian blood, it follows that the latter cannot be considered as the antecedents of the former. To illustrate this point it will be sufficient to compare the size of the two types. The Tr. gambiense as met with in the human blood usually measures about $20 \mu$ in total length, and only exceptionally does it attain a length of 25 to $30 \mu$. Its width is usually from I.4 to $2.0 \mu$; only the divisional forms being slightly wider. On the other hand, the predominating form in $\mathrm{Gl}$. palpalis usually measures 40 to $5 \circ \mu$ in length, and may even be longer, for Gray and Tulloch mention some as being about $100 \mu$ in length. The width of these forms is usually about $2 \mu$, but may at times attain $3 \mu$. In other words the bulk or mass of the tsetse trypanosome is at least twice that of the blood form of Tr. gambiense. This we consider to be important evidence showing that the tsetse trypanosome is not derived from the Tr. gambiense, or for that matter from Tr. Brucei.

Another feature to which attention should be called is the enormously large blepharoplast which Dr. Koch describes in connection with the "female" type of Tr. gambiense as observed in the tsetse fly. Such a large structure has never before been seen in a pathogenic protozoon, but, without questioning the statement, it may be asked whether it is possible to get so enormous a structure in the course of a rapid multiplication of the blood trypanosome in which the blepharoplast is very small and measures only about $0.7 \mu$. On a priori grounds one would expect under these conditions not only trypanosomes which are smaller than the blood forms, but also smaller blepharoplasts than such as are found in the blood forms. In our material, derived from $\mathrm{Gl}$. palpalis, no trypanosomes could be found with blepharoplasts approaching in size that given by Dr. Koch.

Under No. 2.-The presence of trypanosomes in large numbers in tsetses which presumably have never fed upon animals is a fact which argues strongly against a possible relationship to Tr. gambiense or Tr. Brucei. We are informed by Dr. Gray that a greater proportion of the flies examined contained no traces of blood in their intestinal tracts, and this also seems to be the case in Dr. Koch's 
observations. It is very significant, as Dr. Gray writes, that flies captured on the uninhabited island of Kimmi should show 8 per cent infected, whereas those taken along the shore at Entebbe, where there is an abundant opportunity for infection from man, should show only 2 per cent of infections. Dr. Koch's exceptional observation of $\mathbf{1 7 . 4}$ per cent of infected tsetses in Luengeratal, where few antelopes, but many sheep and goats, were present, is hardly explainable by the supposition that they are derived from the latter. It would be well to study the stomach trypanosomes of tsetses in regions where trypanosomiases are rare or absent.

Under No. 3.-As pointed out above, Gray and Tulloch repeatedly met with batches of flies which, after feeding on infected monkeys, failed to show trypanosomes, although other lots fed on the same animals, under identical conditions, gave a small number of positive findings. Their total of to per cent of infection in flies which were thus fed is insufficient evidence to show that they are derived from the trypanosomes in the blood' of the monkeys. The further fact that the monkeys contained but very few trypanosomes, about one per six fields, whereas the flies examined 24 hours after feeding contained enormous numbers, is not explainable on the supposition that these have resulted from the multiplication of the scanty parasites present in the blood which the flies had sucked up. The time was altogether too short for such multiplication to have taken place. On the contrary, it is evidence that shows that the flies were infected at the time of their capture. The fact that Dr. Koch failed to find the trypanosomes in tsetses which fed on richly infected cattle, and obtained but few trypanosomes in flies which fed on oxen and mules, goes simply to show that the mammalian trypanosomes do not multiply in the tsetses. It does not necessitate recourse to the assumption of the existence of some unknown stage of the mammalian trypanosome which is especially adapted or necessary for multiplication in the fly.

Under No. 4.-The failure to infect monkeys by injecting enormous numbers of tsetse trypanosomes, as noted by Gray and Tulloch, and the similar failure of Dr. Koch to infect rats, may be taken as crucial evidence of the non-identity of those flagellates with $T r$. gambiense and Tr. Brucei. Actively growing cultures of $T r$. Brucei 
and $T r$. Lewisi almost invariably infect when injected into animals and a like result should be obtained if the tsetse trypanosomes, which are present in large number and in active multiplication in the fly, have any relation to the pathogenic forms.

The negative experiments referred to cannot be explained satisfactorily in any other way than that the pathogenic trypanosomes which were sucked up died out in a short time in the stomach of the flies. In mosquitoes which are allowed to feed upon a rat infected with $T r$. Brucei, the latter organism may be seen alive for 24 to 36 hours and as long as it is alive such contents will infect mice and rats. After this period, Tr. Brucei cannot be found in the contents, and infection cannot be brought about in animals, notwithstanding the presence of flagellates which, as shown in the previous paper, are the result of a natural infection.

Under No. 5.-The presence of flagellates in the stomachs of tsetses presents a striking analogy to the presence of like forms in the gut of the mosquito. In the case of the latter we have conclusively shown that 15 per cent of the captured or "wild" mosquitoes have a flagellate infection with Herpetomonas, Crithidia, and possibly other species. It would obviously be false logic if we were to examine mosquitoes, after allowing them to feed on rats infected with Tr. Lewisi or Tr. Brucei, and finding flagellates present were to declare that they were multiplication forms of these two wellknown species of trypanosomes. And yet such a conclusion would be fully as justifiable as the assumption that the forms met with in the tsetse were derived from either Tr. Brucei or Tr. gambiense. There is every reason to believe that not one, but several species of trypanosomes, in no wise related to the pathogenic forms, may find their natural habitat in the stomach of the tsetse flies. This is certainly true for the mosquito, and it is not far-fetched to hold that the latter is not the only one in this regard.

The question naturally arises: If the trypanosome in $\mathrm{Gl}$. palpalis is not Tr. gambiense and that in Gl. fusca and Gl. morsitans is not Tr. Brucei, what are they then? No answer to this can be given other than that they are probably harmless non-pathogenic forms. That they are "cultural" forms, there can be no question, and in view of the success of cultivating the mosquito flagellates, 
it is quite certain that the tsetse trypanosome can be readily cultivated in vitro. Such cultures may be expected to show identically the same form as that seen in the fly, and, moreover, such cultures, as seen from the experiments with flies, will be found to have no action on ordinary experimental animals.

As to the origin of these flagellates we can only say that we know as yet but too little of the distribution of trypanosomes in nature to arrive at any definite supposition. If it is assumed that the infected mosquitoes ( 15 per cent) and infected tsetses (2-I 7 per cent) derive their parasites from the blood of animals, it follows that a much larger per cent of these insects must feed, at least once, on animal blood. In other words, there are at least as many uninfected animals bitten, as those which are infected and from which the insects supposedly derive their infection. This would mean that more than 30 per cent of the biting insects, as they live in nature, feed at least once on the blood of some animal. It would seem, on $a$ priori grounds, that this number is altogether excessive, and yet no proof to the contrary can be offered. Notwithstanding this, it is almost as reasonable to believe that only a very small percentage of biting insects ever feed on blood; and that such trypanosomes as they contain are derived from plant juices, stagnant waters, etc. The objection that flagellates have never been observed in such fluids is not a serious one. A careful search may yet reveal the parent form of the trypanosomes which are present in the alimentary tract of ordinary flies, tsetses, mosquitoes, and even in various larval forms. Their presence in the larvæ of mosquitoes, in butterflies, etc., certainly indicates that insects may develop a flagellate infection of the alimentary tract without ever having fed on the blood of infected animals.

THE TRYPANOSOMES OF GLOSSINA PALPALIS.

As indicated in the beginning, it was possible through the kindness of Dr. Gray to stain and examine smears from the intestinal contents of six tsetse flies (Gl. palpalis). These flies were all caught on the lake shore at Entebbe, and after being brought to the laboratory were at once dissected. A little fresh blood serum was added to the intestinal contents of those flies in which trypanosomes were 
found to be present, and from this mixture slide preparations were made. The smears were stained by a modified RomanowskyNocht method, which will be described in a subsequent publication.

A study of these preparations showed: first, that we had to deal with what has already been designated as cultural forms, in distinction from what might be called the blood form of trypanosomes; second, that considerable diversity of type existed, sufficiently so as to justify the opinion that two, and possibly more, species of trypanosomes were present in the specimens examined. Our previous work with cultures of bird trypanosomes made it evident that two very unlike forms may still belong to the same species (as in the case of $T r$. avium), and consequently, in studying the tsetse trypanosomes, this fact was taken into consideration at the outset.

The preparations from Fly No. 9 were particularly rich in trypanosomes which stained quite well, and were hence adapted for a careful examination. Apparently only one species of trypanosome was present in this fly, although under two very unlike forms. The difference, however, is no greater than in the case of $T r$. avium already referred to. Moreover, these two forms correspond very well to the male and female types described by Dr. Koch. In honor of Dr. Gray, who first studied this species, it is here designated as

TRYPANOSOMA GRAYI, N. SP.

As mentioned, this species occurs in $\mathrm{Gl}$. palpalis under two forms. The first of these corresponds to the free-swimming or spirochetelike form of $T r$. avium, and bears considerable resemblance to the

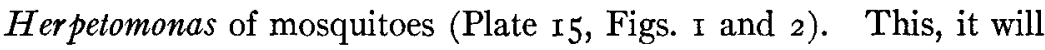
be seen, corresponds to the second or "male" type of Dr. Koch. The body, which is very slender and scarcely stains blue, measures 18-29 $\mu$ in length and from 0.6 to $1.0 \mu$ in width. It is provided with a long, free whip, which is as long as, or longer than, the body proper. The total length, flagellum included, is from $3^{6}$ to $48 \mu$. The anterior end of the body tapers out very gradually along the flagellum, and the existence of a rudimentary undulating membrane may be assumed. The flagellum terminates at, or near, the blepharoplast which is a conspicuous, deeply stained, perfectly round body lying immediately anterior to the nucleus and of about the 
same diameter $(0.6 \mu)$ as the body of the cell. Immediately posterior to the blepharoplast is the very prominent, heavily stained, compact nucleus. This is markedly rod-shaped and measures from 2.3 to $3.3 \mu$ in length by 0.5 to $0.6 \mu$ in width. There is a faint indication of the presence of a diplosome, posterior to the nucleus. The posterior end is sharply pointed; the distance from the tip to the nucleus is from one and one-half to two times the length of the nucleus; i. e., 4.6 to $8 \mu$. No observations have been made regarding the divisional forms of this type which was quite numerous in the preparations.

The second form corresponds in general to the "female" type described by Dr. Koch, though, as will be seen, the blepharoplast is much smaller than as stated by him. In the adult or full-grown stage it may be characterized as a wide and long, ribbon-like form. The plasma is granular, stains a deep blue, and may show colorless globules-a very common occurrence in cultural trypanosomes. The anterior end terminates in a very short flagellum, 2 to $6 \mu$ long. The flagellum is continued along the side of the cell for about twothirds of its length and forms the border or support of a prominent and very wavy undulating membrane; and finally terminates in the achromic zone surrounding the blepharoplast. The latter is usually immediately anterior to the nucleus, though at times it may be lateral or even posterior. In form it is a short oval about $0.4 \times 0.8 \mu$ and is placed transversely. It should be noted that the form designated by Dr. Koch as that of Tr. gambiense has a blepharoplast which is $\mathrm{I} .5 \times 2.5 \mu$. In none of the preparations studied, which, as stated, are derived from $\mathrm{Gl}$. palpalis, and therefore should contain $T r$. gambiense, if the views hitherto published be correct, has anything been found which approaches this so unusually large blepharoplast. In fact, the dimensions given exceed those of the nuclei of our trypanosomes, as can be readily determined from the accompanying photographs.

The nucleus is somewhat round, about $2 \mu$ in diameter, and does not stain as solidly as that of the type just described, but instead shows a granular structure. It lies at a distance from the blunt or square posterior end corresponding to about one-third the length of the cell. The pronounced square-cut end may possibly be due 
to initial involution changes, since some forms have a sharp posterior end. A body, probably corresponding to the acrosome or diplosome which we have described in Tr. Laverani and in the mosquito Herpetomonas, may often be seen near the nucleus, usually between this and the blepharoplast (Plate 16, Figs. 3 and 5). This body is about $3^{\mu}$ long and stains unevenly. Possibly it may correspond to the large body which Dr. Koch designates as a blepharoplast.

This type of trypanosome is subject to considerable variation in size. The largest individuals which we have seen are about $5 \circ \mu$ long by $2.0-2.5^{\mu}$ wide. Smaller forms of one-half to one-third this length are also present. In such the free flagellum is relatively longer than in the full-sized individuals $(7 \mu)$. It may make up onehalf or even more of the total body length. Very young forms are shown in Plate $1_{5}$, Figs. 4 and 6.

Divisional forms of various size are common and, as with other trypanosomes, the division begins with the blepharoplast, thus giving rise to forms with two blepharoplasts, two flagella, and one nucleus (Plate I6, Figs. I and 2). In the more advanced stage the nucleus divides, and then the body proper. The division is very unequal, as has been shown by Gray and Tulloch (Plate I6, Fig. I). Under exceptional conditions, which it is difficult to define, the division of the nucleus may precede that of the blepharoplast (Plate I5, Figs. 3 and 5). In such cases, according to Dr. Koch, the number of nuclei may be two, four, or eight. We have never noted more than two.

Degeneration forms are also met with (Plate I5 $_{5}$ Fig. 5). As can be seen from the photograph, such cells present a swollen appearance, and may show two nuclei with but one blepharoplast. As the result of plasmolytic changes, the protoplasm is shrunken from the ends, and, moreover, becomes banded. It is possible that the consecutive division of the nucleus without that of the blepharoplast, as just mentioned, is the result of similar degenerative changes, rather than a fertilization of the "female" cell, as supposed by Dr. Koch.

OTHER TRYPANOSOMES IN GLOSSINA.

A study of the preparations from the other five flies showed that in only two (Nos. 7 and 8) did the form correspond sufficiently to 
justify the belief that those flies contained the above organism. Both types were present; the narrow or "male" type being identical with that of No. 9. The wide, or "female," form presented slight differences, such as a more pointed posterior extremity and less marked or less wavy undulating membrane, but such variations may be expected from cultures of different age and from different media, as must necessarily obtain in each fly. The Tr. Grayi may therefore be looked upon as the most common form of trypanosome in the tsetse. It constitutes Type $\mathbf{I}$.

Type 2.-A slightly different type from the preceding was met with in Fly No. 10. The narrow or "male" form possessed a longer and wider body, and, in addition, the nucleus, instead of being long and cylindrical, was round or oval (Plate 17 , Fig. I). The more common, or "female," form varied in length from about $I_{5}$ to 35 , and even $5 \circ \mu$. Its width rarely exceeded I. $5 \mu$. The nucleus, unlike that of Tr. Grayi, was elongated and oval in form, but its relative position in the cell was about the same.' The blepharoplast was usually posterior to the nucleus, in varying position, either very near the tip or near the nucleus; at times it was anterior to the same. The flagellum extended close along the body, usually without any marked wavy bends, and terminated as a short free whip 3 to $4 \mu$ long. The posterior end was usually very sharply pointed (Plate $\mathrm{I} 7$, Fig. 2 ).

Type 3.-This was present in Fly No. 6 in rather small numbers. It is markedly different from any of the other forms (Plate I 7, Figs. 3 and 4 ). The "male" form is very much like that of Tr. Grayi, but apparently it is wider and has a much longer free whip. Thr latter is considerably longer than the body, which measures about I2 $\mu$ in length and $\mathrm{r} .5 \mu$ in width. The blepharoplast is anterior or lateral to the nucleus, which is oval or rod-shaped. The "female" forms which stained a deep blue were all short, the body being $12-15$, or at most $20 \mu \mathrm{long}$, and $0.5^{-2.0 \mu}$ wide. The free flagellum measured $5^{-15} \mu$. This form, it may be added, is the only one which might possibly be regarded as having some relation to Tr. gambiense.

Type 4.-Another type quite unlike the others was found in Fly No. 5. Notwithstanding a most careful search nothing similar 
to the "male" forms described in connection with the other types could be found. Instead two very different forms were present. One of these stained a very light blue, and its body tapered along the flagellum almost to its tip. This form usually measured 40-50 $\mu$ in length and $1.5^{-2.0 \mu}$ in width. Although the body was wavy and snakelike, the undulating membrane did not show the wavy folds as in Type I. The nucleus was cylindrical, as in Type 2, but unlike that of the latter, it was quite near, within $3 \mu$, of the posterior end, which was usually truncated, though at times it terminated in a sharp point. The oval blepharoplast was anterior to the nucleus.

This form, it should be noted, corresponds to the measurements which Dr. Koch gives for the male form of Tr. Brucei.

Associated with this form was another which stained a deep blue and had a very granular plasma. Its total length measures from 33 to $45 \mu$. Its widest part $(3 \mu)$ is about $6 \mu$ from the posterior end, which tapers to a blunt point. Anteriorly the body constricts rather suddenly and becomes gradually narrower until it reaches the tip of the flagellum. At times a short free whip is present. The nucleus is roundish or oval and is made up of loose chromatin particles. The blepharoplast may be either anterior or posterior to the nucleus (Plate I7, Fig.5).

Agglutination groups of the narrow form were met with, and in these the whips were directed outward. Occasionally two cells were found agglutinated, the posterior ends overlapping in exactly the same manner, as we have shown in our photographs of Tr. avium. $^{\mathrm{I}}$ Divisional forms corresponding closely to those of Type I (Plate I6, Fig. I) were present. Degenerative forms, as seen from the rounding up of the posterior end, were quite common.

In separating the forms observed into the several types described above it is not intended to convey the impression that these are distinct species. Whether they are distinct species or mere variations of one species cannot be settled at present by mere microscopic examination. Considerable variation in form is met with in a few of the cultures of trypanosomes which we have studied. This is notably true of Crithidia, which, while it usually appears in the

${ }^{x}$ See Jour. Infect. Dis., 1905, 2, Plate 1o, Figs. 3 and 4. 
typical short, truncated form, at times, under certain conditions of the medium or temperature, gives rise to long, spindle-shaped cells which could easily be supposed to represent a distinct species. Hence, extreme care must be exercised in interpreting the forms observed in the alimentary tract of insects. The conditions in no two insects are necessarily alike, and the kind and amount of food recently taken is not without influence on the size and number of the organisms present.

The purpose in presenting these observations is to call attention to what we have designated as the cultural characteristics of trypanosomes. The presence of such forms in the stomach or intestines means that these organisms can be cultivated artificially, and, that being the case, it is incumbent upon every investigator engaged in studying the living forms to obtain pure cultures of the organisms in question before drawing conclusions as to their supposed relationship to pathogenic trypanosomes or to intracellular parasites. As in bacteriological work, where the essential requirement of pure cultures is demanded, so also in this class of protozoal work the necessity of pure cultures can no longer be ignored. In our opinion, too many hasty conclusions have been drawn already from the mere morphological study of the flagellates observed in sanguivora, such as leeches, lice, mosquitoes, and flies.

The conclusion, in brief, to be drawn from this study is that the trypanosomes of the tsetse flies have nothing to do with the pathogenic Tr. gambiense or Tr. Brucei, and that they are mere harmless parasites of the alimentary tract analogous to those known to exist in mosquitoes.

As this paper is going to press our attention has been called to the results of a similar investigation carried out by Professor E. A. Minchin. The original is not accessible, but from an abstract it appears that he regards the tsetse fly as a mere carrier and not as a host of the trypanosome of sleeping sirkness, and the flagellates which are present in its stomach he considers as parasite peculiar to the fly and in no wise related to the mammalian trypanosomes. 
PLATE I 5 .

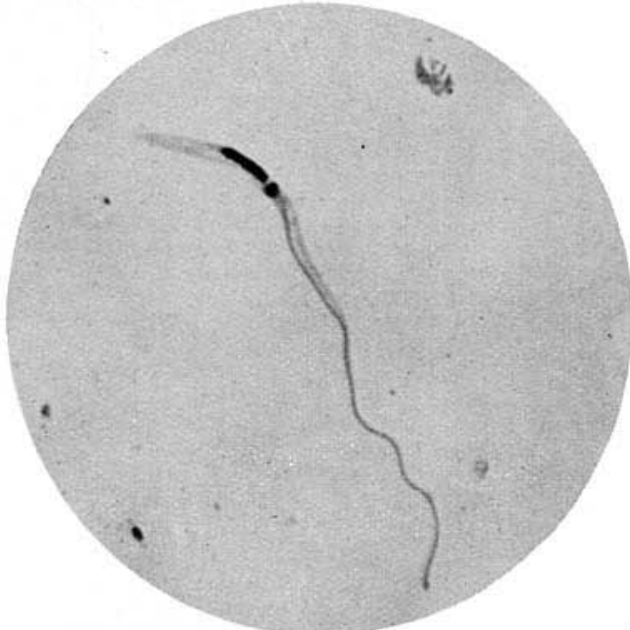

FIG. I.

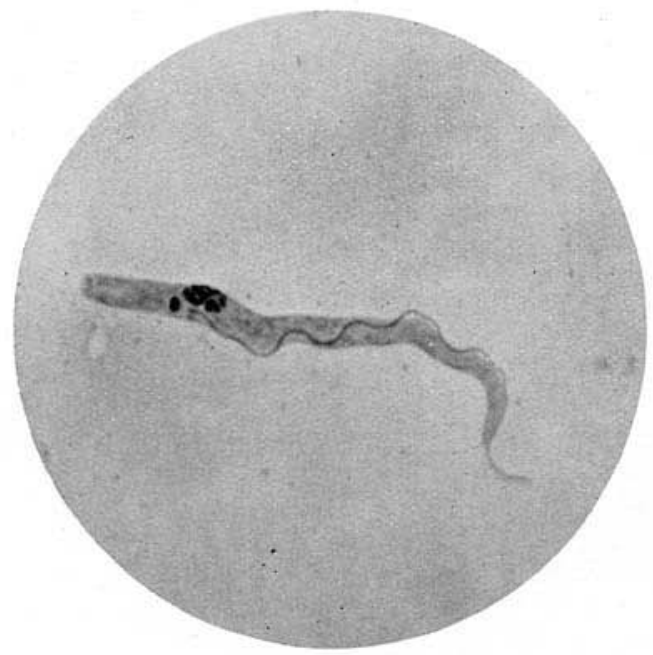

FIG. 3 .

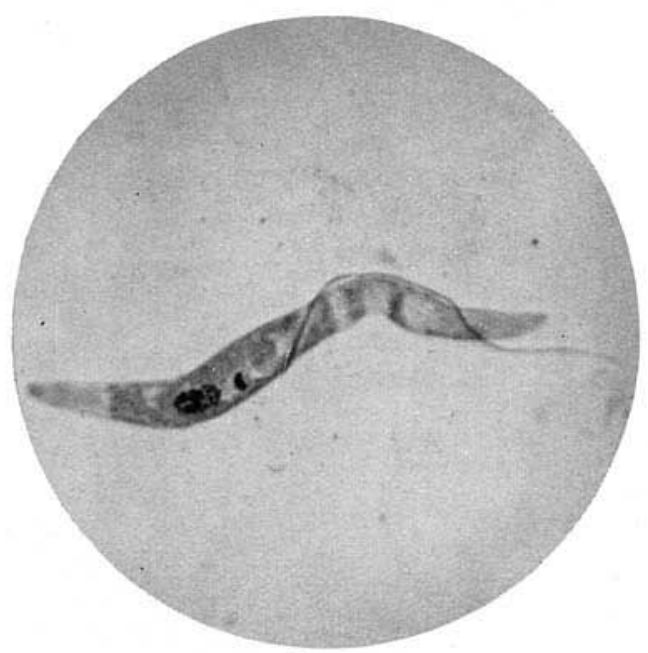

FIG. 5 .

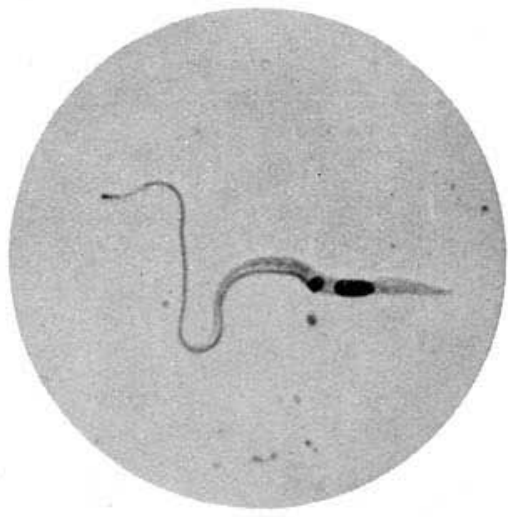

FIG. 2.

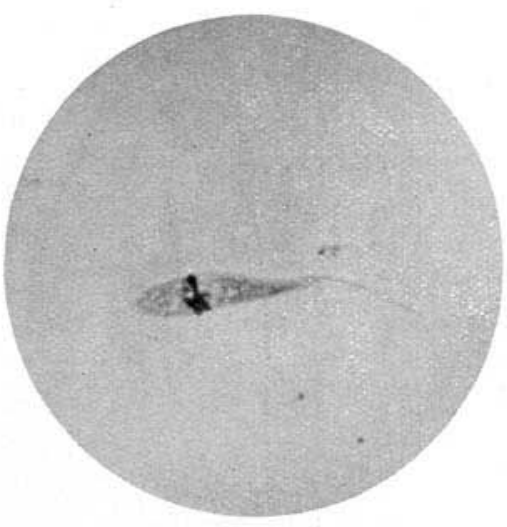

FIG. 4.

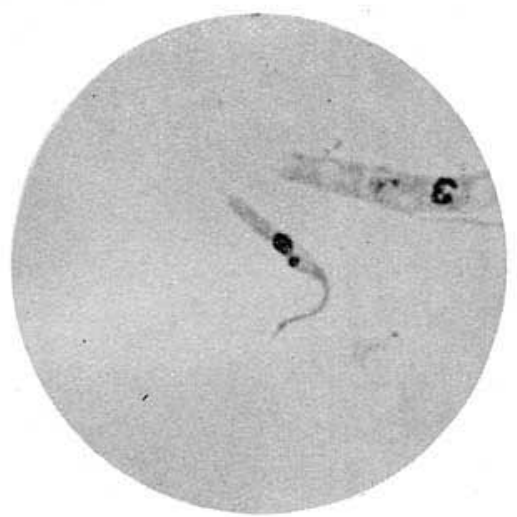

FIG. 6. 
PLATE 16.
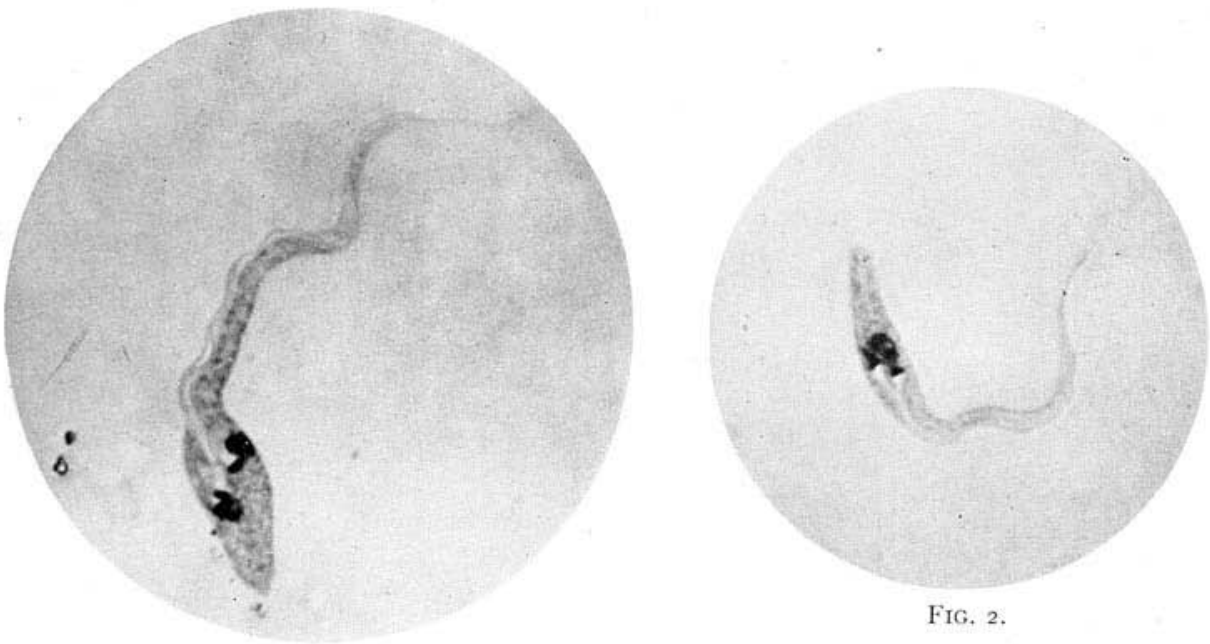

FIG, 2.

FIG. I.

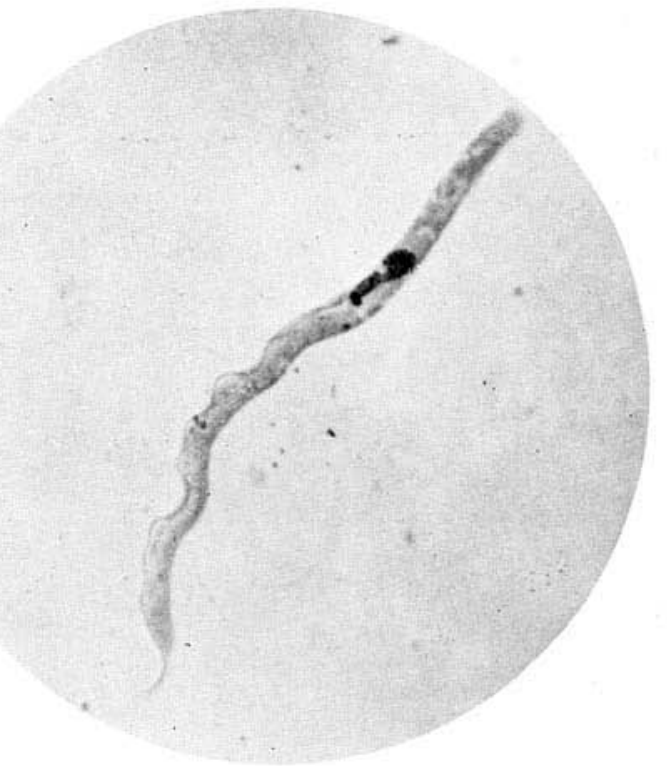

FIG. 5 .
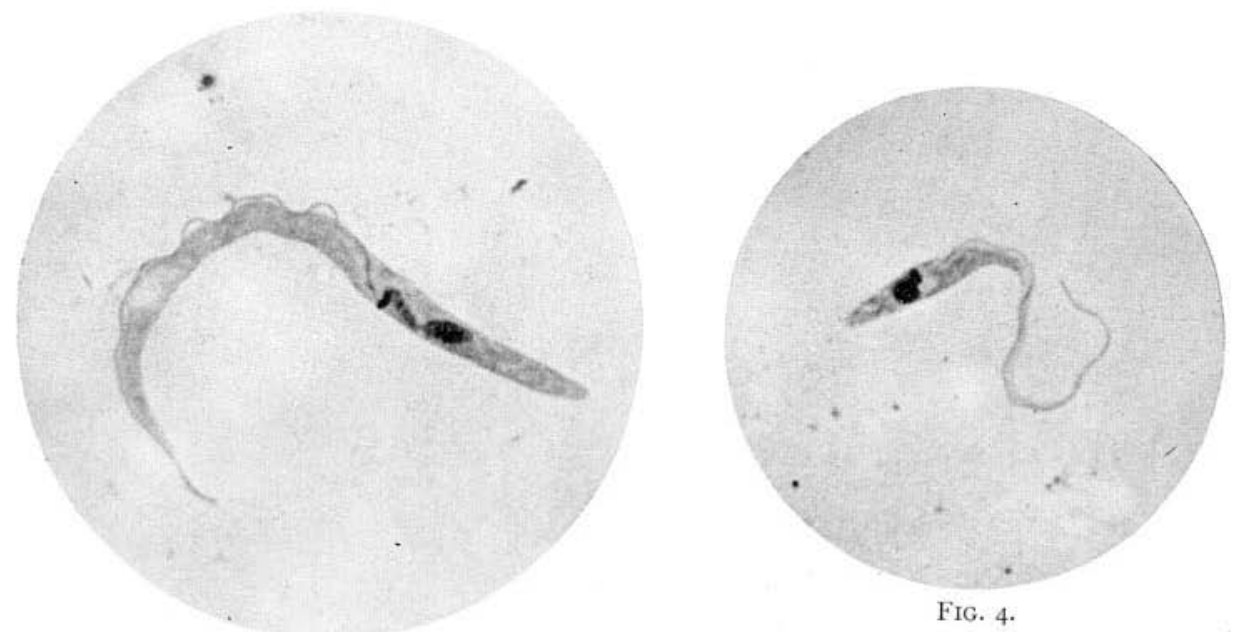

FIG. 4 .

FIG. 3. 
PLATE 17.
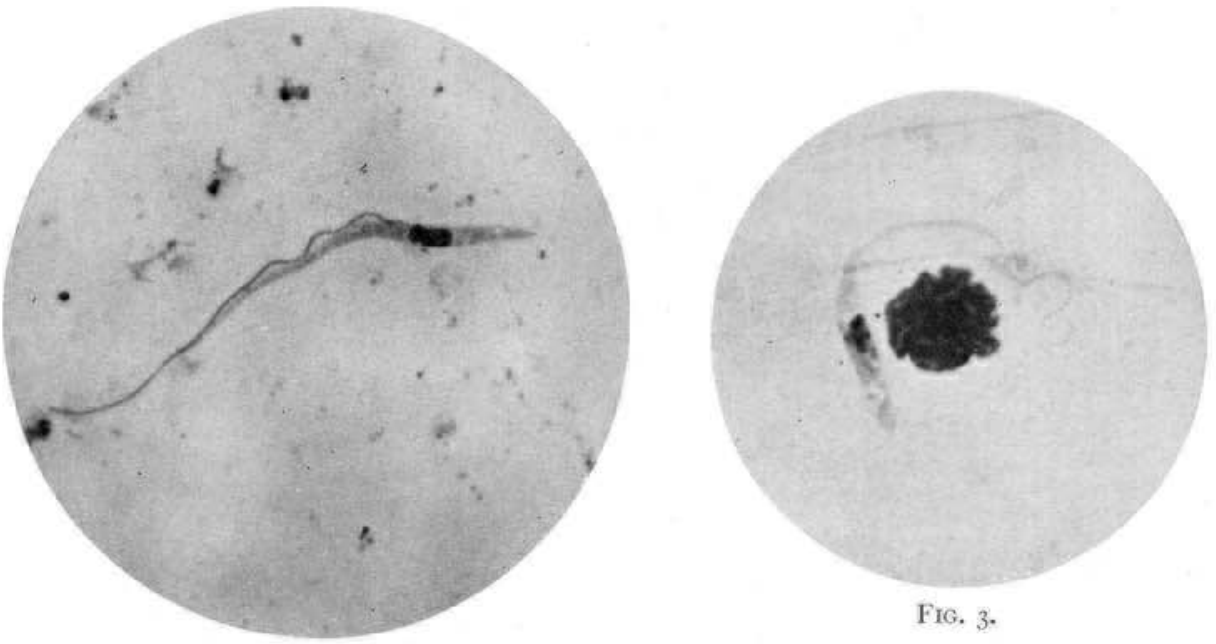

Fig. 3.

FIG, I.

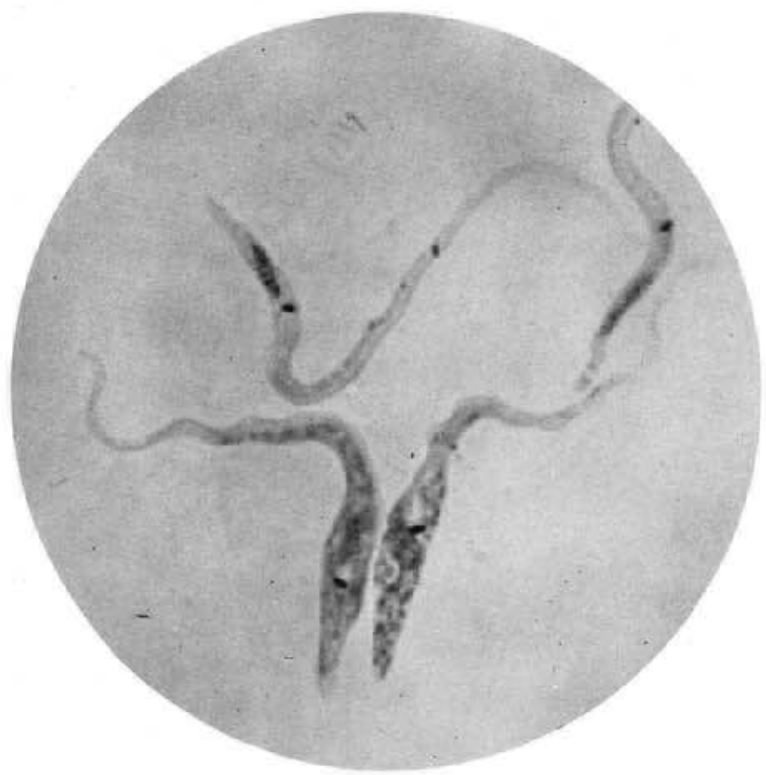

FIG. 5 .

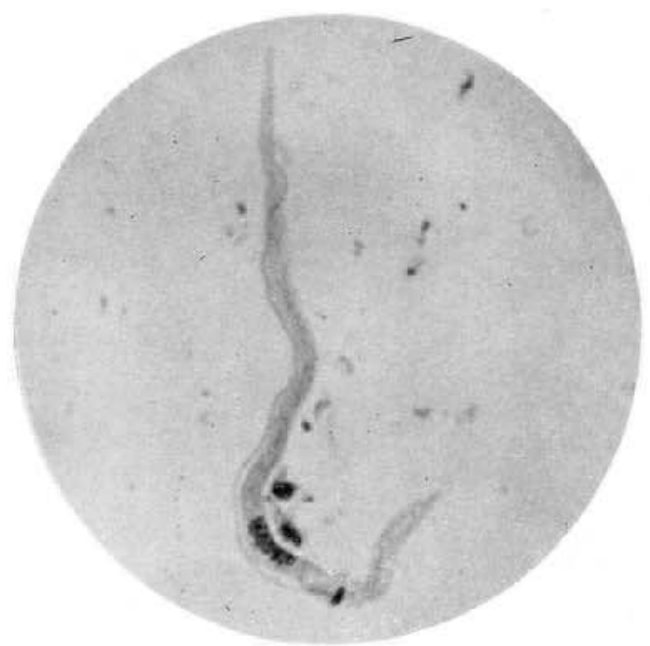

FIG. 2.

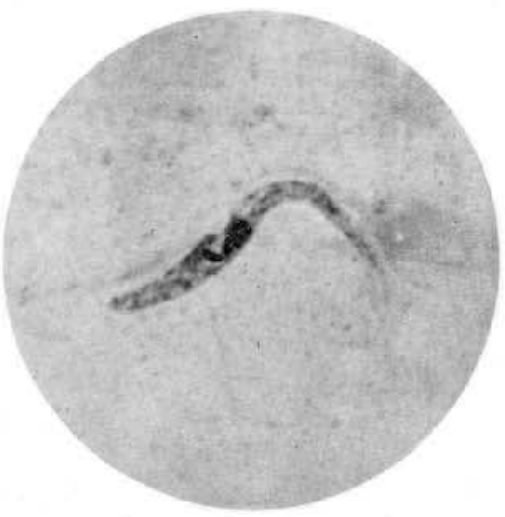

FIG. 4. 


\section{EXPLANATION OF PLATES.}

The accompanying photographs are all taken at the uniform magnification of 1,500 diameters, and, as such, they are directly comparable with those of other cultural trypanosomes previously given.

PLATE 15. TRYPANOSOMA GRAYI, N. SP.

All the photographs are taken from Tsetse No. 9, except Fig. 4, which is from Fly No. 7 .

FIg. I.-A long, narrow, or "male" form. Note the free flagellum as long as the body; also the long cylindrical compact nucleus and the prominent round blepharoplast.

FIG. 2.-A similar form from the same preparation. Indication of a diplosome posterior to the nucleus.

Fig. 3.-A broad, blue-staining, or "female" form. Shows the nucleus in process of division and anterior to the oval blepharoplast; also the square posterior end.

FIG. 4.-A young form of the same type.

FIG. 5.-A degeneration of the same. Note the increase in width, also presence of two nuclei with but one blepharoplast. The darker portion, in parts broken up into bands, represents the blue-stained plasma which shows plasmolytic changes with consequent retraction from the cell-wall.

FIG. 6.-A very young form with the square posterior end of a very large trypanosome projecting into the field.

\section{PLATE 16. TRYPANOSOMA GRAYI.}

Figs. I and 2 are from Fly No. 7 , the others are from No. 9 .

FIG. I.-A large "female" form in process of division; it shows two nuclei and two blepharoplasts. Note the unequal longitudinal division.

FIg. 2.-A smaller form, showing earlier stage of division, one nucleus and two blepharoplasts.

FIG. 3.-A very long form, showing the very prominent undulating membrane and the short free whip. Note the presence of the diplosome between the nucleus and the blepharoplast.

FIG. 4.-A young form of the same with a relatively long free whip.

Fig. 5.-A very long form, showing the diplosome between the nucleus and the blepharoplast; also prominent undulating membrane and short free whip; truncated posterior end.

PLATE 17. SHOWING TYPES 2, 3, AND 4.

FIG. I.-A slender "male" form of Type 2 in Fly No. Io. Note the thick oblong nucleus and anterior to this the large round blepharoplast.

FIG. 2.-A long form of the blue-stained, or "female," cell from the same preparation as the preceding. Note the cylindrical, granular nucleus, and posterior to this the rod-shaped, transversely placed, blepharoplast; also the very short free whip.

FIG. 3.-A slender "male" form of Type 3 from Fly No. 6. Note the very long free flagellum.

FIG. 4.-The usual form of the blue-stained, or "female," of Type 3 from the same preparation as the preceding. The free flagellum can scarcely be seen.

FIG. 5- - The two forms of Type 4 from Fly No. 5. Below are two deeply bluestained, highly granular, or "female," forms. Above can be seen the faintly stained, narrow form, with cylindrical nucleus lying close to the posterior end. 